\title{
Klotho expression is a prerequisite for proper muscle stem cell function and regeneration of skeletal muscle
}

\author{
Hellen E. Ahrens ${ }^{1 \dagger}$, Judith Huettemeister ${ }^{1,2 \dagger}$, Manuel Schmidt ${ }^{1}$, Christoph Kaether ${ }^{1}$ and Julia von Maltzahn ${ }^{1 *}$ (D)
}

\begin{abstract}
Background: Klotho is a well-known anti-aging hormone, which serves as a suppressor of aging through a variety of mechanisms. Aging of skeletal muscle is concomitant with a decrease in muscle stem cell function resulting in impaired regeneration.

Methods: Here we investigate the functional role of the anti-aging hormone Klotho for muscle stem cell function after cardiotoxin-induced injury of skeletal muscle using a klotho hypomorphic mouse line, which is characterized by a premature aging phenotype. Furthermore, we perform floating single myofiber cultures with their adjacent muscle stem cells to investigate the interplay between canonical Wnt signaling and Klotho function.

Results: We demonstrate that muscle stem cell numbers are significantly decreased in klotho hypomorphic mice. Furthermore, we show that muscle stem cell function is also severely impaired upon loss of klotho expression, in culture and during regeneration in vivo. Moreover, we demonstrate that addition of recombinant Klotho protein inhibits aberrant excessive Wnt signaling in aged muscle stem cells thereby restoring their functionality.

Conclusions: The anti-aging hormone Klotho counteracts aberrant canonical Wnt signaling in muscle stem cells and might be one of the naturally occurring inhibitors of canonical Wht signaling in skeletal muscle.
\end{abstract}

Keywords: Skeletal muscle, Regeneration, Klotho, Myogenesis, Muscle stem cell, Aging, Canonical Wnt signaling, Wnt3a

\section{Background}

Skeletal muscle is one of the tissues with the highest ability to regenerate [1]. Muscle stem cells, also named satellite cells, are a prerequisite for functional regeneration $[2,3]$. Under resting conditions they are quiescent and located under the basal lamina [4]. After injury, muscle stem cells become activated, proliferate, and differentiate to form new myotubes [5]. Muscle stem cell functionality can be affected by intrinsic changes in the cells themselves and changes in the environment-extrinsic changes [1, 4]. Diseases such as muscular dystrophy and other conditions such as natural aging are major factors influencing muscle stem cell function [6-8]. However, during aging, the regenerative capacity of skeletal muscle decreases dramatically [7, 9-12]. Furthermore, muscle stem cell number and functionality decline

\footnotetext{
* Correspondence: julia.vonmaltzahn@leibniz-fli.de

${ }^{+}$Hellen E. Ahrens and Judith Huettemeister contributed equally to this work. ${ }^{1}$ Leibniz-Institute on Aging - Fritz-Lipmann-Institute, Beutenbergstrasse 11, 07745 Jena, Germany

Full list of author information is available at the end of the article
}

with age caused by intrinsic and extrinsic changes [8]. Interestingly, several studies demonstrated constant upregulation of a number of developmental signaling pathways such as JAK/STAT and Hox signaling in aged muscle stem cells $[7,11,12]$. Additionally it is known that systemic factors and changes in the muscle stem cell niche can influence regeneration of skeletal muscle in the aged [10].

Klotho is a well-known anti-aging gene coding for the aklotho protein, which serves as a suppressor of aging through a variety of mechanisms [13]. Two transcripts can arise from the klotho gene through alternative splicing. Thereby, a membrane protein (mKlotho) and a secreted protein are generated, of which the latter one is lacking the transmembrane domain [14]. Furthermore, mKlotho can be cleaved and shedded by secretases, and this form of klotho is referred to as the soluble klotho. The secreted protein and the soluble klotho are here both referred to as sKlotho. Importantly, the different forms of klotho (soluble and membrane bound) inhibit 
or activate different signaling pathways [13, 14]. mKlotho serves as an obligate co-receptor for fibroblast growth factor 23 (FGF23) in many tissues, e.g., the kidney [15]. There, FGF23 signaling inactivates 1,25-dihydroxyvitamin D3 synthesis and inhibits phosphate reabsorption via ion channel $\mathrm{NaPi} 2 \mathrm{a}$, thus regulating mineral homeostasis [16]. The soluble form of Klotho is mostly shed into the circulation where it interacts with different signaling pathways in the target organs. For instance, sKlotho is known to inhibit insulin/IGF-1 signaling [17]. Furthermore it was demonstrated that sKlotho can inhibit $\mathrm{Wnt} / \beta$-catenin and TGF- $\beta$ signaling and might serve as a potential tumor suppressor [18].

Interestingly, serum levels of soluble Klotho decline with age in mice and men $[19,20]$. This is in line with reports on klotho hypomorphic mice ( $\Delta$ Klotho), a well-established model of premature aging [21]. Those mice are genetically characterized by an insertional mutation in the promoter of the klotho gene leading to a severe hypomorphic variant through reduced transcription of the klotho gene [21]. Mice, which are homozygous for this mutation develop multiple signs of aging including reduced life span, kyphosis, osteoporosis, and arteriosclerosis. Klotho hypomorphic mice are indistinguishable from their wild type littermates until weaning (p21, postnatal day 21) but then rapidly develop a premature aging phenotype with reduced growth, kyphosis, and osteoporosis. Around postnatal day 40 (p40), the aging phenotype is fully developed [21]. Conversely, klotho overexpression leads to an increased lifespan in mice by up to $20-30 \%$ [22]. Klotho is predominantly expressed in the kidney, the parathyroid gland, and the cerebral choroid plexus, but also in other organs including skeletal muscle [23].

So far, little is known about the expression and function of klotho in the skeletal muscle. mRNA transcript was detected in lysates from the whole skeletal muscle [21] while the cell type/cell types expressing klotho and its function are still unknown. A study by Phelps et al. in 2013 demonstrated that muscle strength and running endurance are significantly decreased in klotho hypomorphic mice when compared to wildtype littermates [24]. So far, the underlying cause of this decline in muscle strength still needs to be identified. The process of muscle regeneration is fine-tuned and depending on muscle stem cells, which are affected by intrinsic factors in muscle stem cells themselves as well as by systemic effects and factors coming from the stem cell niche [1]. One of the signaling pathways affecting regeneration of skeletal muscle is canonical Wnt signaling described to be increased in aged skeletal muscle [25]. sKlotho is a known inhibitor of canonical Wnt signaling. Therefore, we investigated the effect of Klotho on regeneration of the skeletal muscle, muscle stem cell function, and the interplay between canonical Wnt signaling and sKlotho in muscle stem cells.

We show that klotho hypomorphic mice display disturbed muscle stem cell function as well as reduced regenerative capacity. Furthermore, we identify sKlotho as one of the modulators of muscle stem cell function and thereby regeneration of skeletal muscle, potentially by inhibiting aberrant canonical Wnt signaling, e.g., in the context of aging.

\section{Methods \\ Mice}

Klotho deficient ( $\Delta$ Klotho) mice used in this study were the original hybrid klotho mutant mice backcrossed to $129 \mathrm{~Sv}$ inbred mice for more than nine generations as described previously [21]. Wildtype and heterozygous littermates served as controls. The C57BL/6J mice used for myofiber culture experiments were obtained from Janvier. Mice were kept in an SPF facility with food and water ad libitum and a fixed 12-h day/night light cycle. All animal experiments were performed in accordance with the German Animal Welfare Act and approved by the responsible local authority of Thuringia (TLV), TVA no.: 03-11/14.

\section{Muscle injury}

Mice were anesthetised with isoflurane. The right hind limb was shaved and disinfected before $50 \mu \mathrm{l}$ cardiotoxin ( $10 \mu \mathrm{M}$ in $0.9 \% \mathrm{NaCl}$, Sigma) were injected into the tibialis anterior muscle using a 29 gauge needle as described previously [26]. Analgesics (meloxicam $1 \mathrm{mg} / \mathrm{kg}$ body weight) were applied for 3 days. Animals were sacrificed 10 or 21 days after muscle injury.

\section{Immunofluorescence and immunoblot analyses}

Tibialis anterior (TA) and extensor digitorum longum (EDL) muscles were isolated, embedded in OCT (Tissue $\mathrm{Tec}$ ) containing $10 \%$ sucrose and snap-frozen in liquid nitrogen.

Immunofluorescence on thin cryosections $(12 \mu \mathrm{m})$ was performed after fixation with 2\% PFA, permeabilisation (0.1\% TritonX100, $0.1 \mathrm{M}$ glycine in phosphate buffered saline (PBS)) and blocking for $1 \mathrm{~h}$ at $\mathrm{RT}$ in $2.5 \%$ mouse-on-mouse (M.O.M.) blocking solution (Vector labs) in PBS. Primary myoblasts, differentiated myotubes, and myofibers were fixed with $2 \%$ PFA, permeabilised, and blocked with $5 \%$ horse serum in PBS for $1 \mathrm{~h}$ at RT. The following primary antibodies were used: anti-Ki67 (rabbit, 1:1000, ab15580, AbCam), anti-Laminin (rabbit, 1:1000, Sigma), anti-MyoD (rabbit, 1:250, Santa Cruz; rat, 1:200 Merck), anti-Pax7 (mouse IgG1, undiluted, Developmental Studies Hybridoma bank (DSHB)), anti-myogenin (mouse IgG1, F5D, undiluted, DSHB), anti-myosin heavy chain (MHC) (mouse IgG2b, MF20, undiluted, DSHB), 
anti-MHC type IIa (mouse IgG1, SC-71, undiluted, DSHB), anti-MHC type IIx (mouse IgM, 6HI, undiluted, DSHB), anti-MHC type IIb (mouse IgM, BF-F3, undiluted, DSHB), anti-developmental MHC (devMHC) (mouse IgG1, 1:500, BF-45, DSHB), and anti-CD68 (rat, 1:500, Biorad MCA1957). No fixation with PFA was used for devMHC stainings.

The following secondary antibodies were used: anti-rabbit IgG (Alexa-Fluor 488), anti-rabbit IgG (Alexa-Fluor 647), anti-mouse IgG1 (Alexa-Fluor 546 and Alexa 488), anti-mouse IgG2b (Alexa-Fluor 488), and anti-mouse IgM (Alexa-Fluor 488 and Alexa-Fluor 546) (all obtained from Life Technologies, all diluted 1:1000). Nuclei were counterstained with DAPI (1:5000) before mounting with Permafluor (Thermo Scientific).

Immunoblot analyses were performed using the following antibodies: MuRF1/2/3 (rabbit, 1:1000, ab172479, AbCam), Atrogin/Fbxo32 (rabbit, 1:1000, ab168372, AbCam), non-phospho (active) $\beta$-Catenin (Ser33/37/ Thr41) (D13A1) (rabbit, 1:1000, 8814S, Cell Signaling), phospho (inactive) $\beta$-Catenin (Ser33/37/Thr41) (rabbit, 1:1000, 9561S, Cell Signaling), Klotho (goat, 1:2000, AF1819, R\&D Systems), Tubulin (mouse, 1:500, T9026, Sigma-Aldrich), GAPDH (G-9) (mouse, 1:200, sc-365062, Santa Cruz), peroxidase goat anti-rabbit (1:1000, P0448, Dako), peroxidase rabbit anti-goat (1:1000, P0449, Dako), and peroxidase goat anti-mouse (1:1500, P0447, Dako), including Pierce $^{\text {Ta }}$ ECL Western blotting substrate (32106, Thermo Scientific) and Immobilon ${ }^{\text {TM }}$ Western chemiluminescent HRP substrate (WBKLS0500, Millipore). Images were acquired with a MyECL Imager (Thermo Scientific).

\section{Histochemistry}

Hematoxylin-Eosin (H\&E) stainings of TA cross-sections were performed automatically with the Leica stainer XL as described earlier [26]. Sirius Red, Oil RedO, and Alizarin Red stainings were performed as described earlier [26].

\section{Muscle stem cell isolation}

Complete hind limb musculature was minced and incubated with collagenase B (2.5 g/ml, Roche) and dispase II ( $1 \mathrm{~g} / \mathrm{ml}$, Roche) for $30 \mathrm{~min}$ at $37{ }^{\circ} \mathrm{C}$. The digested muscles were further homogenized by trituration and filtered through $74-\mu \mathrm{m}$ cell filters. Isolated cells were pelleted at $450 \times g$ and gently resuspended in $500 \mu$ l Magnetic-activated cell sorting (MACS) buffer (0.5\% BSA, 2 mM EDTA in PBS) followed by addition of $60-\mu$ microbeads coupled to monoclonal antibodies against non-target cells according to instructions provided by the manufacturer (Satellite Cell Isolation Kit, Miltenyi Biotec $\mathrm{GmbH}$ ). After incubation for $30 \mathrm{~min}$ on ice, cells were filtered through a $50-\mu \mathrm{m}$ filter and loaded on a MACS column (Miltenyi Biotec). The flow-through was collected, washed, and plated on collagen-coated 10-cm cell culture dishes. Staining for Pax7 was performed to check for purity of the isolated cells.

\section{Cell culture}

Primary myoblasts were kept in Ham's F-10 Nutrient Mix supplemented with $20 \%$ fetal bovine serum, $2 \%$ penicillin/ streptomycin, and $2.5 \mathrm{ng} / \mathrm{ml}$ bFGF (Gibco) at $37{ }^{\circ} \mathrm{C}$ and $5 \%$ CO2. For the proliferation assay, cells were washed in PBS, trypsinized, and seeded onto collagen-coated 24-well plates (25,000 cells per well). After $48 \mathrm{~h}$, cells were fixed with $2 \%$ PFA for 5 min followed by subsequent immunofluorescence staining. For differentiation assays cells (33,000 cells per 24-well) were cultured for 3 or 5 days in DMEM containing $2 \%$ horse serum and $2 \%$ penicillin/ streptomycin. Concentration of cell culture supernatants was performed using Amicon columns with a cut-off of $30 \mathrm{kDA}$ as described earlier [27].

\section{EDL single fiber culture}

Single myofibers were isolated from EDL muscle by digestion in DMEM with $0.2 \%$ collagenase (from Clostridium hystolyticum, Sigma) and cultured in DMEM supplemented with 20\% FBS and 1\% chick embryo extract (US biological) as described previously [27].

Myofibers of old (22-24 months) and young (24 months) C57BL6/J mice were incubated with recombinant human soluble Klotho protein $(100 \mathrm{ng} / \mathrm{ml}$, tebu-bio, Cat\# enz-369), recombinant mouse Wnt3a (100 ng/ml, R\&D Systems, \# P27467), recombinant mouse Dkk1 (100 ng/ml, R\&D Systems, \# O54908), or different combinations of those.

\section{RNA isolation, cDNA synthesis, and quantitative real-time PCR (qRT-PCR)}

Total RNA was isolated from freshly isolated or cultured (72 h) EDL-derived myofibers and kidney samples by using the peqGOLD TriFast (VWR) according to the manufacturer's instructions. cDNA was synthesized with the iScript cDNA Synthesis Kit (BioRad) according to manufacturer's instructions. Quantitative real-time PCR was performed in technical triplicates using the iQ SYBR Green Supermix (BioRad) on the Mx3000P qPCR System (Agilent Technologies). The following program was used: $3 \mathrm{~min}$ at $95{ }^{\circ} \mathrm{C} ; 40$ cycles of $95{ }^{\circ} \mathrm{C}$ for $15 \mathrm{~s}$, followed by $58{ }^{\circ} \mathrm{C}$ for $15 \mathrm{~s}$, and $72{ }^{\circ} \mathrm{C}$ for $30 \mathrm{~s}$; and a final cycle of $95{ }^{\circ} \mathrm{C}$ for $15 \mathrm{~s}$, followed by $58{ }^{\circ} \mathrm{C}$ for $15 \mathrm{~s}$, and $95{ }^{\circ} \mathrm{C}$ for $30 \mathrm{~s}$. qPCR primers for mouse alpha-Klotho detection were purchased from Genecopoeia (Product ID MQP031135). For normalization, we used GADPH primers with the following sequence $\left(5^{\prime}-3^{\prime}\right)$ : GAPDH_USP (forward): ATGCCAGTGAGCTTCCC and GAPDH_DSP (reverse) CATCACCATCTTCCAGGAGC. We calculated relative mRNA expression values with the $\Delta \Delta \mathrm{Ct}$ 
method: $\Delta \mathrm{Ct}=\mathrm{Ct} \quad(\alpha \mathrm{Klotho})-\mathrm{Ct} \quad(\mathrm{GAPDH})$, relative expression $=2^{(-\Delta \mathrm{Ct})}$.

\section{Digital image acquisition and processing}

Images were acquired with the Axio Imager and Axio Observer.Z1 (Carl Zeiss). Immunofluorescence images from whole muscle cross-sections were generated by using the tile mode at 20-fold magnification with the ZEN software (Carl Zeiss). Representative image details were selected from whole cross-section tiled images. The minimal fiber feret of myofibers was determined as the closest distance between two parallel tangents of the fiber [28].

\section{Statistical analysis}

Data were analyzed with the GraphPad Prism software. Statistical significance was determined by an unpaired, one-tailed Student's $t$ test. Results are presented as mean \pm standard error of the mean. The calculated $P$ values are shown as $* *$, and $* *$ which represent $P<0.05, \quad P<0.01, \quad$ and $P<0.001$, respectively.

\section{Results}

Muscle stem cell numbers are reduced in $\Delta$ Klotho mice Several reports suggested that stem cell function in different organs is perturbed in $\Delta K$ lotho mice, e.g., adipogenic stem cells [29]. We first asked whether the muscle stem function is also affected in $\Delta$ Klotho mice. Therefore, we investigated if the number of muscle stem cells shows differences when comparing muscles from $\Delta$ Klotho mice and their littermate controls. Indeed, we found a reduction in the number of muscle stem cells in $\Delta$ Klotho mice at p56 and p14 while numbers were comparable at p21, a time point when skeletal muscle matures (Fig. 1a-d). Furthermore, we found an age-dependent difference in myofiber size between $\Delta K$ lotho and control mice (Additional file 1: Figure $\mathrm{S} 1 \mathrm{~A}-\mathrm{C})$. Of note, at p14, the size of myofibers of $\Delta$ Klotho and control littermates did not differ. With age,
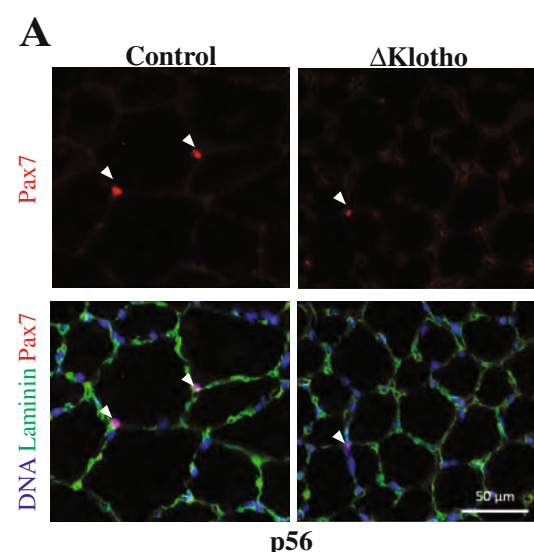

C

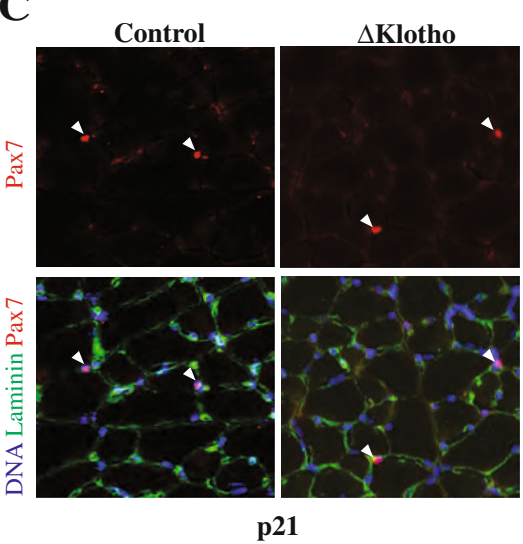

$\mathbf{B}$

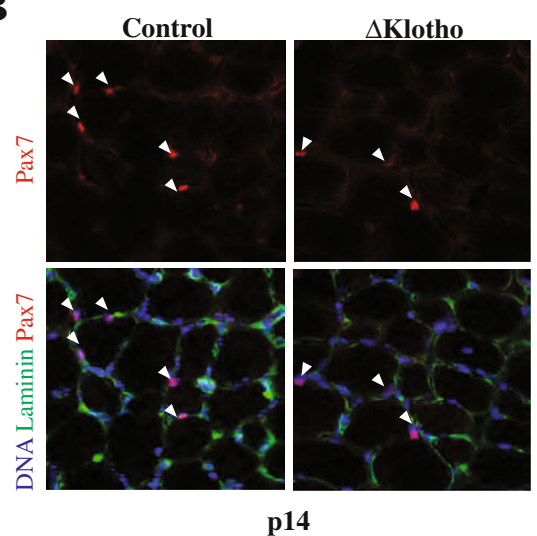

D

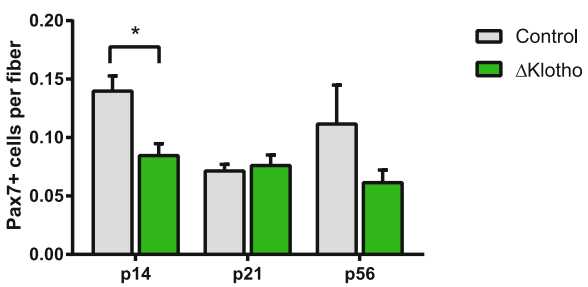

Fig. 1 Muscle stem cell numbers are reduced in $\Delta$ Klotho mice. a-c Representative immunofluorescence images of TA muscle cross-sections from $\Delta$ Klotho and control mice stained for DAPI (DNA, blue) and Laminin (green), and Pax7 (red) at a p56, b p14, and $\mathbf{c}$ p21. Scale bar $=50 \mu \mathrm{m}$. $\mathbf{d}$ Quantification of Pax7-positive SCs on whole TA cross-sections from $\Delta$ Klotho and control mice at p14, p21, and p56. $n=3$ mice per genotype and time point. All data are presented as means \pm SEM. ${ }^{*} p<0.05$ 
the average fiber feret in muscles of control mice increased at p21 and p56 as expected. In contrast, the average fiber feret from $\Delta$ Klotho mice did not increase with age (Additional file 1: Figure S1B). Accordingly, the size of tibialis anterior muscle cross sectional area demonstrated differences at p56 between $\Delta$ Klotho and control littermates (Additional file 1: Figure S1C). These age-dependent changes in myofiber size could be either due to a defect in muscle growth or due to a premature aging phenotype which would be concomitant with increased expression of atrophy markers, e.g., MuRF1-3 and Fbxo32/Atrogin/MAFbx [30-33]. Therefore, we investigated the expression of the ubiquitin ligases MuRF1-3 and Fbxo32/Atrogin/MAFbx in $\Delta$ Klotho and age-matched control littermates (Additional file 1: Figure S1D). Here, we found that expression of all ubiquitin ligases was increased in $\Delta$ Klotho muscles compared to age-matched littermate controls (Additional file 1: Figure S1E). Furthermore, TA muscles are lighter in $\Delta$ Klotho mice (Additional file 1: Figure S1F) also when the muscle weight is normalized to the tibia length (Additional file 1: Figure S1G). This is suggesting that muscles from $\Delta$ Klotho mice are more likely undergoing a sarcopenia-like muscle wasting than a developmental defect, while also a combination of both is possible. When analyzing the fiber type distribution in EDL muscles between $\Delta$ Klotho and control littermates with an age of p56, we did not observe any differences (Additional file 2: Figure $\mathrm{S} 2 \mathrm{~A}-\mathrm{C}$ ). The reduced myofiber size in $\Delta \mathrm{K}$ lotho mice at an age of p56 concomitant with the increased expression of the ubiquitin ligases (MuRF1-3 and Fbxo32/Atrogin/MAFbx) in muscles from $\Delta$ Klotho mice suggest that $\Delta$ Klotho mice display an age-dependent reduction in muscle mass, which is reminiscent of sarcopenia. This decreased muscle mass is accompanied by reduced numbers of muscle stem cells, which might lead to impaired regeneration of skeletal muscle.

\section{Regeneration of skeletal muscle is severely impaired in $\Delta$ Klotho mice}

Impaired muscle stem cell function generally coincides with decreased regenerative capacity of skeletal muscle $[4,34]$. To analyze the role of klotho during regeneration, we injured the tibialis anterior muscles of $\Delta$ Klotho and control mice at an age of p50 and analyzed the regeneration 10 days after injury (Fig. 2a). Intriguingly, we already observed obvious impairments in the regeneration process in muscles from $\Delta$ Klotho mice on the macroscopic level (Fig. 2b). Muscles from control littermates after 10 days of regeneration showed the expected degree of regeneration (d10) (compare [34]). In contrast, $\Delta$ Klotho mice exhibited signs of fibrosis, calcification, and scarring suggesting major impairments in regeneration, confirmed by histological analyses (Fig. 2c). To quantify regeneration, we measured the minimal fiber feret as a measure of myofiber size. Thereby, we found significantly smaller myofibers after $\mathrm{d} 10$ of regeneration in $\Delta$ Klotho mice (control $23.58 \pm 1.08 \mu \mathrm{m}$ vs. $\Delta$ Klotho $16.70 \pm 0.71 \mu \mathrm{m})$ (Fig. 2d, e). This suggests either impaired differentiation of muscle stem cells or loss of self-renewal capacity thereby limiting the number of stem cells available for formation and growth of newly formed fibers. To further investigate the differentiation process in vivo, we quantified the number of devMHC (developmental myosin heavy chain) positive myofibers (Fig. 2d, f), a well-known marker for newly formed myofibers. Importantly, during maturation of myofibers, expression of devMHC decreases [35]. We found that $82 \%$ of myofibers in $\triangle$ Klotho mice still expressed devMHC while only $47 \%$ of myofibers in the control littermates were positive for devMHC at day 10 after CTX injury. This indicates that differentiation/maturation of myofibers is delayed/inhibited in $\Delta$ Klotho muscles (Fig. 2d, f). Muscle stem cells can divide asymmetrically during the regeneration process thereby generating progenitor cells to build new myofibers or self-renew to replenish the pool of stem cells [25]. To investigate the self-renewal potential of muscle stem cells, we counted the number of muscle stem cells (Pax7 positive) at day 10 after injury. Here, we found a dramatic reduction of $69 \%$ in muscle stem cell numbers in $\Delta$ Klotho muscles (control $0.200 \pm 0.016$ Pax $7+$ cells/fiber vs. $\Delta$ Klotho $0.062 \pm 0.033$ Pax7+ cells/fiber; Fig. $2 \mathrm{~g}$ ) while numbers of macrophages were increased in $\Delta$ Klotho mice (Fig. $2 \mathrm{~h}$ ). These results suggest that differentiation/maturation of newly formed myofibers and self-renewal of muscle stem cells are severely compromised.

To further address the self-renewal ability of muscle stem cells in vivo, we investigated regeneration of skeletal muscle 21 days after injury (Fig. 3a), a time point when the regeneration process is nearly completed and muscle stem cells start to become quiescent again [4]. Gross morphological impairments in muscle regeneration were also obvious 21 days after injury in $\Delta$ Klotho mice (Fig. 3b), again showing scarring, aberrant calcification, accumulation of lipids, and signs of fibrosis as observed 10 days after injury (Fig. 2b, c). Histological analyses demonstrated that the regeneration process in $\Delta$ Klotho mice was still strongly impaired compared to littermate controls with noticeably smaller myofibers and increased numbers of mononucleated cells at day 21 after injury (Fig. 3c) suggesting either increased infiltration of immune cells or lack of differentiation of myogenic cells. For quantification of the regeneration process, we measured the minimal fiber feret and found also here a significant decrease $(32 \%)$ in myofiber size in muscles from $\Delta$ Klotho mice compared to littermate controls (control $31.77 \pm 0.97 \mu \mathrm{m}$ vs. $\Delta$ Klotho $21.75 \pm$ 

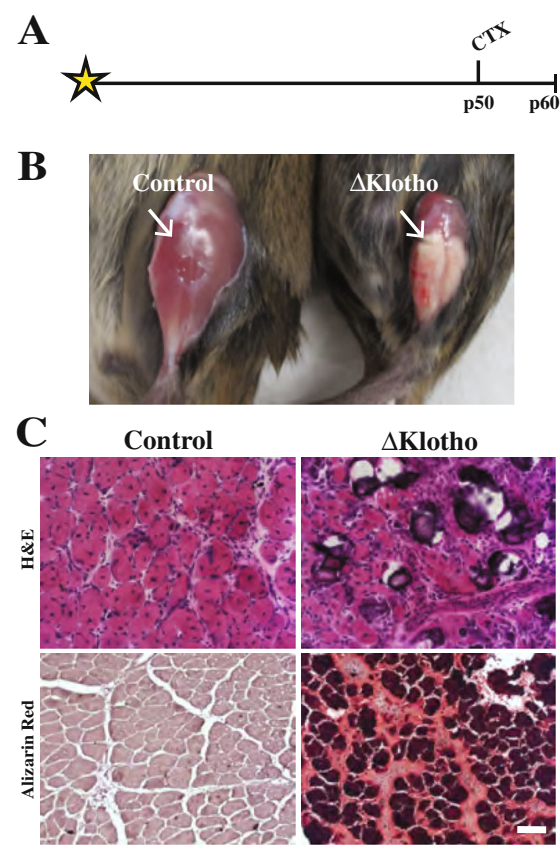

\section{G}

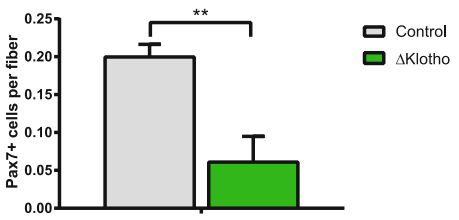

D

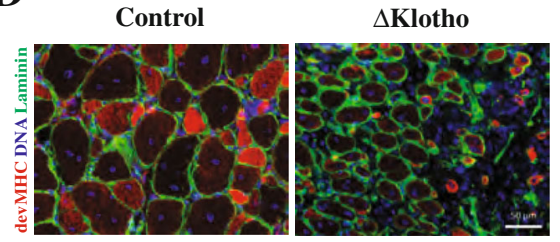

$\mathbf{E}$

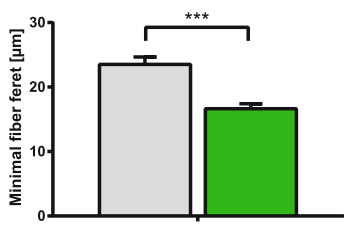

F

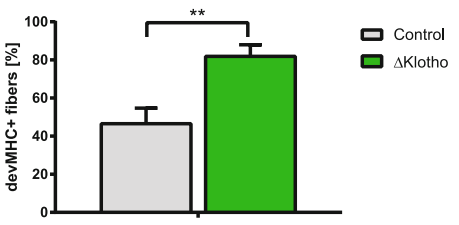

$\mathbf{H}$

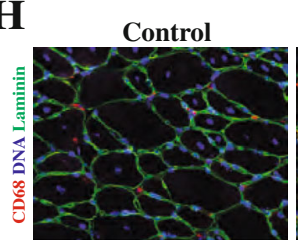

$\Delta$ Klotho

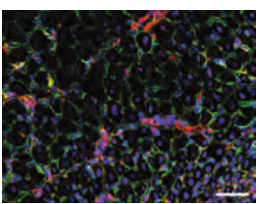

Fig. 2 Early regeneration of skeletal muscle is impaired in $\Delta$ Klotho mice. a The right TA muscle from $\Delta$ Klotho and control mice was injured by injection of cardiotoxin (CTX) at p50 and isolated at p60. b TA muscles (arrows) show extensive fibrosis in $\Delta$ Klotho mice 10 days (d10) after injury. c Representative H\&E stainings and Alizarin Red stainings of TA muscle cross-sections at d10 after injury. Necrotic tissue, fatty vacuoles, and massive cell invasion are visible in $\Delta$ Klotho TA muscles, while control TA muscles show mostly regenerating myofibers. Scale bar $=100 \mu \mathrm{m}$. $\mathbf{d}$ Immunostainings of cross-sections of TA muscles at d10 after injury with antibodies directed against Laminin (green) and developmental (dev) MHC (red), nuclei are stained with DAPI (DNA, blue). Scale bar $=50 \mu \mathrm{m}$. e Minimal fiber feret measured on whole cross-sections from $\Delta$ Klotho and control TA muscles at d10 after injury. ( $\Delta$ Klotho $n=4$ mice, control $n=5$ mice). Proportion of $\mathbf{f}$ devMHC-positive fibers and $\mathbf{g}$ Pax7-positive cells per myofiber on whole cross-sections at d10 after CTX-induced injury ( $\Delta$ Klotho $n=3$ mice, control $n=4$ mice). $\mathbf{h}$ Representative images of stainings for CD68 (in red) and laminin (in green) showing increased infiltration with macrophages in regenerating muscles from $\Delta$ Klotho mice. Scale bar $=50 \mathrm{um}$. All data are presented as means \pm SEM. ${ }^{*} p<0.05,{ }^{* *} p<0.01,{ }^{* * *} p<0.001$

$1.62 \mu \mathrm{m}$; Fig. 3d). To get further insights into the maturation process of newly formed myofibers, we counted the number of devMHC-positive myofibers at day 21 after injury (Fig. 3f). In control muscles, we hardly found any devMHC-positive myofibers as expected and described in the literature [35]. Consistent with our previous observations, the percentage of devMHC myofibers in $\Delta$ Klotho muscles was increased by nearly twofold (control $6.49 \pm 1.73 \%$ vs. $\Delta$ Klotho $12.08 \pm 5.083 \%$; Fig. 3f) suggesting impaired differentiation/maturation of myofibers in $\Delta$ Klotho mice after injury. When we counted the number of Pax7-positive muscle stem cells 21 days after regeneration, we found a dramatic decrease in $\Delta$ Klotho mice compared to littermate controls (60\% difference; Fig. $3 \mathrm{~g}$ ) indicating severe impairments in self-renewal of muscle stem cells when klotho expression is lost. Furthermore, numbers of macrophages were still increased at day 21 after regeneration in $\Delta$ Klotho mice (Fig. 3h).

\section{Loss of mklotho does not affect myogenic proliferation or differentiation}

In the in vivo model, we used the expression of both forms of klotho (mKlotho and sKlotho) which are ubiquitously perturbed. Furthermore, it is not possible to investigate the role of mKlotho independently of systemic effects including reduced serum levels of sKlotho, which arise from loss of klotho expression in the whole body. To gain information about expression of klotho mRNA (detecting mRNA for mKlotho and sKlotho) in myogenic cells, we performed expression analyses of primary myoblasts and myotubes (Fig. 4a). We can detect klotho mRNA in myoblasts (d0), 

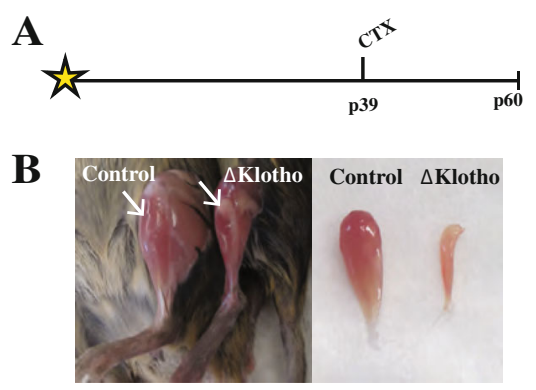

$\mathbf{C}$
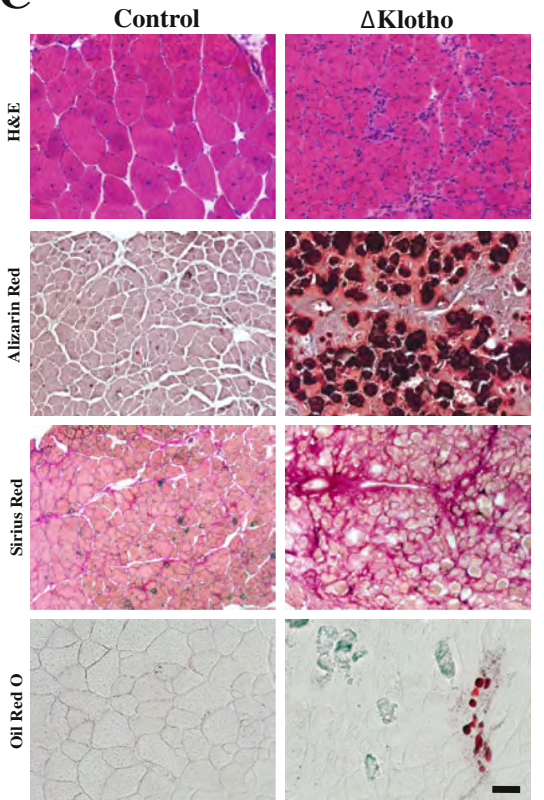

D

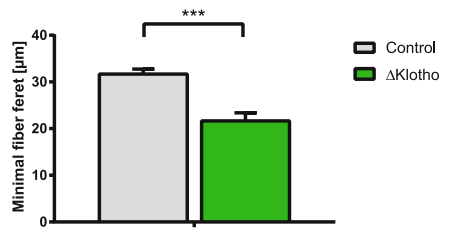

$\mathbf{E}$
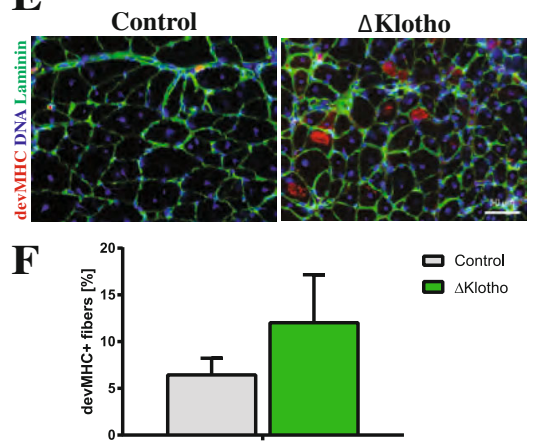

G

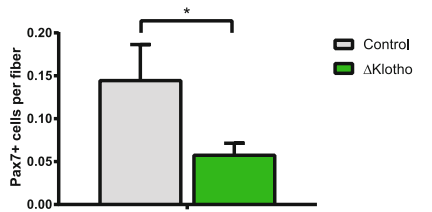

H

Control
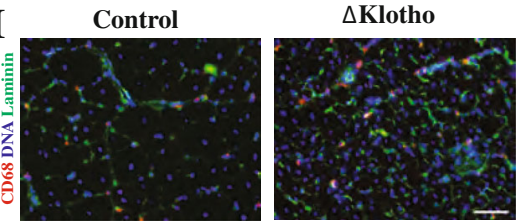

Fig. 3 Late regeneration of skeletal muscle is impaired in $\Delta$ Klotho mice. a The right TA muscle from $\Delta$ Klotho and control mice was injured by injection of cardiotoxin (CTX) at p39 and isolated at p60. b TA muscles (arrows) show signs of impaired regeneration by H\&E staining and extensive fibrosis in $\Delta$ Klotho mice at day 21 after injury (c), but not in control littermates as demonstrated by Sirius Red staining. Alizarin Red marks calcifications, visible in regenerating muscles from $\Delta$ Klotho mice as well as lipid accumulations shown by Oil Red O staining. Scale bar $=100 \mu \mathrm{m}$. $\mathbf{d}$ Minimal fiber feret measured on whole cross-sections of TA muscles from $\Delta$ Klotho and control at d21 after injury. ( $\Delta$ Klotho $n=4$ mice, control $n=4$ mice). e Immunostainings of cross-sections of TA muscles at d21 after injury with antibodies directed against laminin (green) and developmental (dev) MHC (red), nuclei are stained with DAPI (DNA, blue). Scale bar $=50 \mu \mathrm{m}$. Proportion of $(\mathbf{f})$ devMHC-positive fibers ( $\Delta$ Klotho $n=2$ mice, control $n=4 \mathrm{mice}$ ) and (g) Pax7-positive satellite cells per myofiber on whole cross-sections at d21 after CTX-induced injury. ( $\Delta$ Klotho $n=4$ mice, control $n=4$ mice). $\mathbf{h}$ Representative images of stainings for CD68 (in red) and laminin (in green) showing increased infiltration with macrophages in regenerating muscles from $\Delta$ Klotho mice. Scale bar $=50 \mathrm{um}$. All data are presented as means \pm SEM. ${ }^{*} p<0.05,{ }^{* *} p<0.01,{ }^{* * *} p<0.001$

where expression decreases following differentiation suggesting that expression of klotho mRNA is most important in undifferentiated cells. Furthermore, we asked whether myoblasts secrete sKlotho (Fig. 4b, Additional file 3: Figure S3E, F). Indeed, we found that myoblasts isolated from wt animals secrete small amounts of sKlotho (Additional file 3: Figure S3E), this is lost in primary myoblasts from $\Delta$ Klotho mice-as expected (Fig. 4b). We then isolated primary myoblasts from $\Delta$ Klotho and control littermates and analyzed their proliferation and differentiation potential (Fig. 4c-g). Importantly, by using this approach, we exclude systemic factors, e.g., changes in the mineral homeostasis of $\Delta$ Klotho mice and sKlotho secreted by the kidney and transported to the muscle via the blood stream.
Furthermore, we exclude factors from the muscle stem cell niche, e.g., changes in the extracellular matrix. The only difference between the myogenic cells from $\Delta$ Klotho and control mice is the expression of mKlotho and secretion of sKlotho by myoblasts or myotubes themselves. Interestingly, no differences in proliferation (Fig. 4c, d) or differentiation (Fig. 4e-g and Additional file 3: Figure S3 A-D) were observed between myogenic cells isolated from $\Delta$ Klotho and control littermates implying that expression of mKlotho and secretion of sKlotho in myoblasts does not affect their proliferation and differentiation. Therefore, we can conclude that endogenous expression of mKlotho is dispensable for proper proliferation and differentiation of myoblasts. 
A

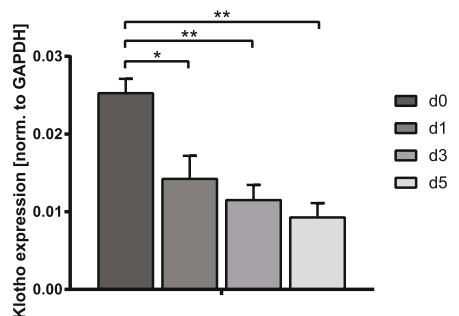

C
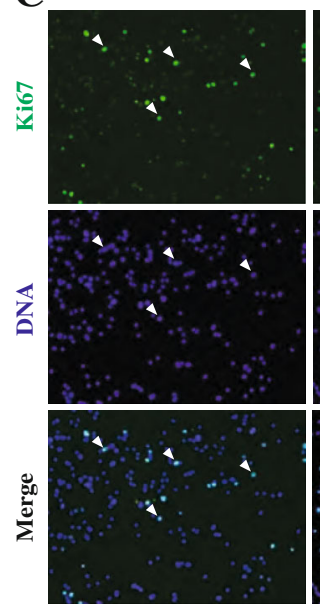

D

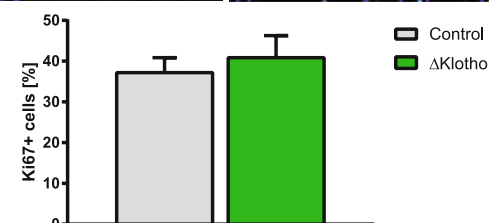

$\mathbf{E}$
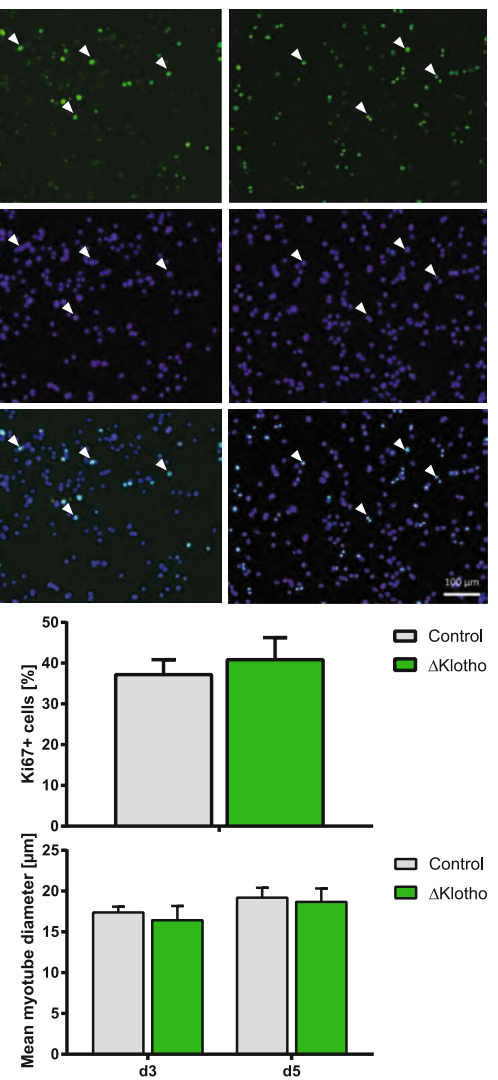

B

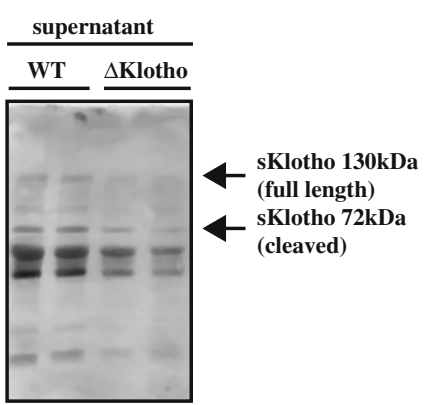

F
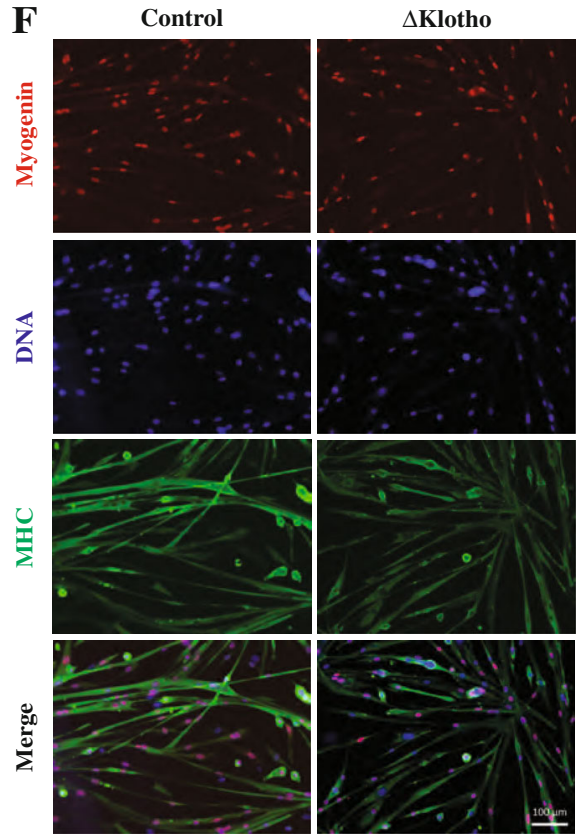

G

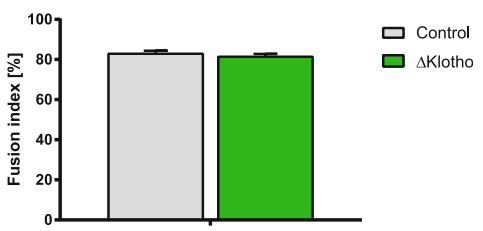

Fig. 4 Proliferation and differentiation are not affected in myoblasts from $\Delta$ Klotho mice in vitro. a aklotho mRNA expression during myogenic differentiation of myoblasts d0, growth medium (myoblasts); d1, day 1 of differentiation (myocytes); $d 3$ and d5, days 3 and 5 of differentiation (myotubes). ( $n=3$ mice per time point). All data are presented as means \pm SEM. ${ }^{*} p<0.05,{ }^{* *} p<0.01$. Expression was normalized to GAPDH. $\mathbf{b}$ Immunoblot analysis showing expression of sKlotho in the supernatant from primary myoblasts isolated from wt mice but not in the supernatant from $\Delta$ Klotho mice. An image of the Ponceau stained membrane can be found in Additional file 3: Figure S3F. c Primary myoblasts from $\Delta K$ Klotho and control mice were immunostained for the proliferation marker Ki67 (green) and DAPI (DNA, blue) after $48 \mathrm{~h}$ of proliferation time in normal culture medium. Arrow heads mark Ki67 positive cells. Scale bar $=100 \mu \mathrm{m}$. d The proportion of Ki67-positive cells was counted in 15 randomly chosen regions of interest per condition ( $\Delta$ Klotho $n=3$ mice, control $n=4$ mice). e The mean myotube diameter was measured in six randomly chosen regions of interest per condition ( $\Delta$ Klotho $n=3$ mice, control $n=4$ mice). $\mathbf{f}$ Representative images of immunostainings from myotubes after 5 days of differentiation. DNA (DAPI, blue), MHC (green), myogenin (red). Scale bar $=100 \mu \mathrm{m}$. $\mathbf{g}$ The fusion index was calculated as the ratio of nuclei in myotubes in relation to the total number of nuclei in 6 randomly chosen regions of interest per condition ( $\Delta$ Klotho $n=3$ mice, control $n=4$ mice). All data are presented as means \pm SEM

\section{Muscle stem cell function is severely impaired in adult} $\Delta$ Klotho mice

To investigate the muscle stem cell function in their endogenous niche but without the influence of systemic factors, we performed single myofiber cultures from
EDL muscles [36]. In this culture system, the muscle stem cells are located in their endogenous niche adjacent to the muscle fiber and can undergo multiple rounds of divisions under controlled culture conditions. Therefore, we first compared the number, activation, and 
A
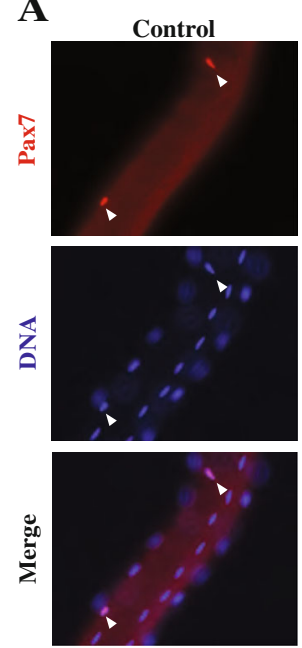

$\mathbf{B}$

Control
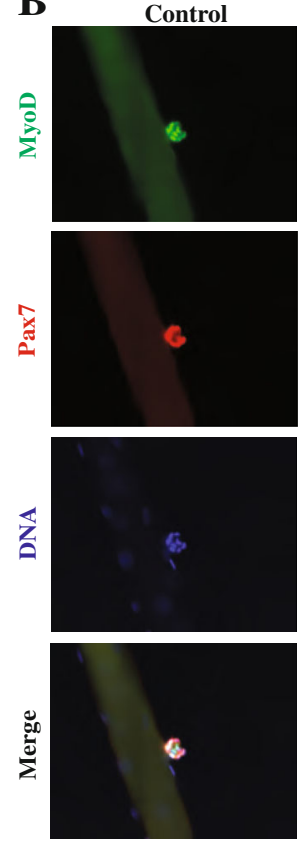
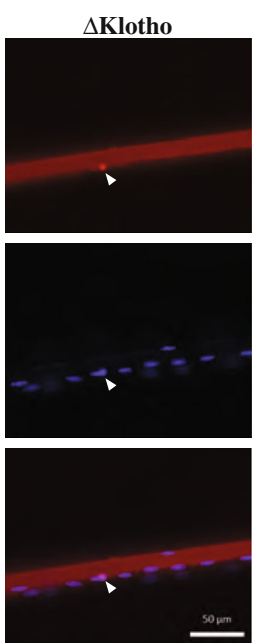

$\Delta$ Klotho
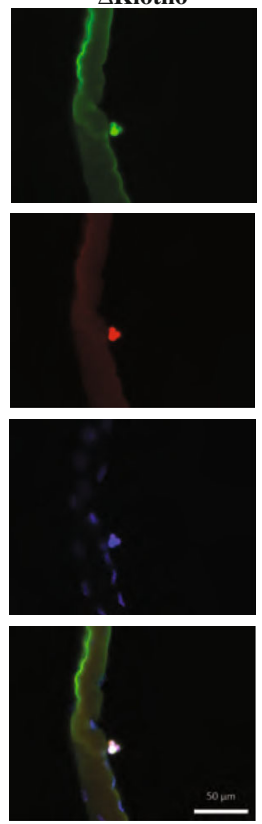

C

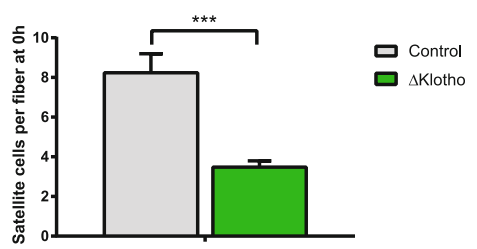

D

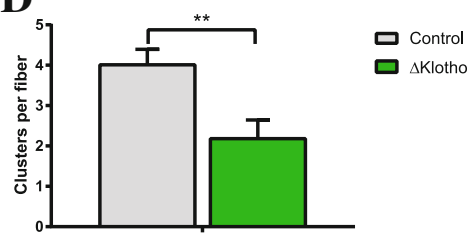

$\mathbf{E}$
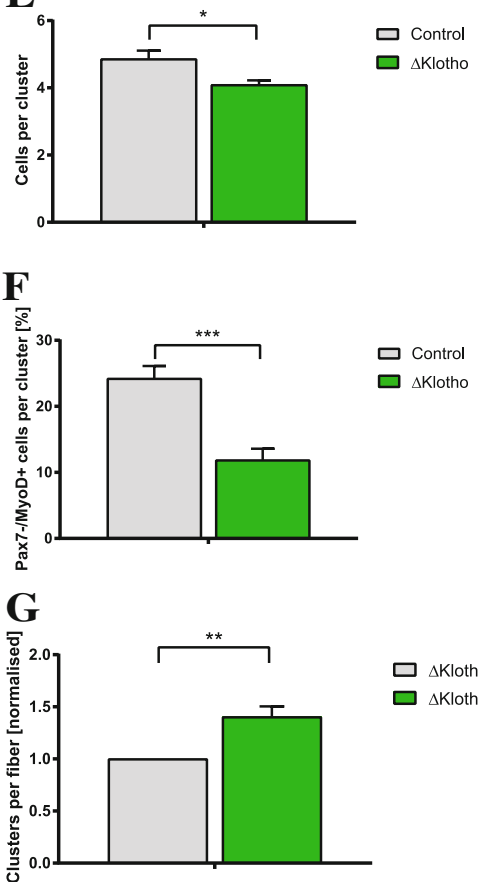

Fig. 5 Muscle stem cell function is impaired in $\Delta$ Klotho mice. a Myofibers with their adjacent muscle stem cells were isolated from EDL muscles of p42 old mice and directly fixed and stained with antibodies directed against Pax7 (red) and DNA (DAPI, blue). Arrows point at Pax7-positive muscle stem cells. Scale bar $=50 \mu \mathrm{m}$. b Representative images of clusters of muscle stem cells on their adjacent EDL myofibers isolated at p42 and cultured for $72 \mathrm{~h}$ and then stained with antibodies directed against MyoD (green), Pax7 (red), and DNA (DAPI, blue). Scale bar $=50 \mu \mathrm{m}$. $\mathbf{c}$ The number of Pax7-positive muscle stem cells per myofiber was quantified from p42 $\Delta$ Klotho and control mice. ( $\Delta$ Klotho $n=5$ mice, control $n=$ 7 mice). $\mathbf{d}$ The number of clusters per myofiber isolated from p42 animals and cultured for $72 \mathrm{~h}$ of culture was enumerated. ( $\Delta$ Klotho $n=5$ mice, control $n=7$ mice). e The number of cells per cluster per myofiber isolated from $\mathrm{p} 42$ animals and cultured for $72 \mathrm{~h}$ was determined. ( $\Delta K \mathrm{Klotho} n$ $=5$ mice, control $n=7$ mice). $\mathbf{f}$ The composition of clusters shows a reduction in the proportion of further differentiated cells (Pax7-/MyoD + ) in $\Delta$ Klotho compared to control mice at p42. ( $\Delta$ Klotho $n=5$ mice, control $n=7$ mice). $\mathbf{g}$ Addition of recombinant sKlotho to fiber cultures from $\Delta$ Klotho mice results in an increase in cluster number per myofiber $(n=3)$. All data are presented as means \pm SEM. ${ }^{*} p<0.05$,

${ }^{* *} p<0.01,{ }^{* * *} p<0.001$

differentiation potential of muscle stem cells of $\Delta$ Klotho and control mice. We observed a dramatic reduction (58\%) in the average number of muscle stem cells per myofiber in $\Delta$ Klotho mice directly after isolation (Fig. $5 \mathrm{a}$, c). Importantly, the reduction of muscle stem cells observed in the EDL was similar to the one observed in the TA muscle (Fig. 1a, d). To investigate the proliferative capacity, we determined the number of clusters formed from a single muscle stem cell. Notably, we also observed a dramatic reduction in the average number of 
clusters per myofiber in $\Delta$ Klotho mice compared to littermate controls (Fig. 5b, d) as well as in the average number of cells per cluster (Fig. 5e). This indicates that proliferation and/or differentiation of muscle stem cells are affected in $\Delta$ Klotho mice. However, we did not observe differences in the activation potential (Additional file 4: Figure S4A). After muscle injury or culture, muscle stem cells are activated resulting in the expression of MyoD and acquisition of a progenitor status. Subsequent downregulation of Pax7 (Pax7-/MyoD+) is followed by differentiation [37, 38]. Indeed, we found a reduction in the percentage of cells, which only express MyoD in clusters from $\Delta$ Klotho mice (Fig. 5f; Additional file 4: Figure S4B-D). This is suggestive of impaired differentiation of muscle stem cells in $\Delta$ Klotho mice. Interestingly, the same loss of differentiation can be seen in muscle stem cells cultured on floating myofibers isolated from aged mice [7]. To investigate if recombinant sKlotho (KL) is able to rescue the decrease in cluster formation observed in $\Delta$ Klotho mice, we applied sKlotho recombinant protein to fiber cultures from $\Delta$ Klotho mice (Fig. 5g). Indeed, addition of sKlotho results in an increase in the number of clusters per fiber of $\Delta$ Klotho mice suggesting that sKlotho and not mKlotho is important for muscle stem cell function.

\section{Wnt3a antagonizes Klotho function in muscle stem cells}

Canonical Wnt is one of the secreted factors, which play an important role in stem cell maintenance and stem cell proliferation [39]. In muscle stem cells, canonical Wnt signaling controls differentiation of muscle stem cells; for instance, it was demonstrated that a switch from Notch signaling to canonical Wnt signaling is a prerequisite for the differentiation of muscle stem cells [25, 40, 41]. Although necessary for differentiation, exogenous induction of canonical Wnt signaling during early regeneration of skeletal muscle leads to premature differentiation of progenitor cells thereby depleting the muscle stem cell pool [40]. Furthermore, it was described that addition of canonical Wnt3a to adult skeletal muscle leads to increased amounts of connective tissue resulting in a skeletal muscle, which resembles aged skeletal muscle after regeneration. sKlotho is a known inhibitor of canonical Wnt signaling [42]. The KL1 domain of sKlotho was recently mapped as the interaction domain of sKlotho with Wnt3a [43]. This evidence prompted us to speculate if sKlotho could be an inhibitor of canonical Wnt signaling in muscle stem cells.

Therefore, we first evaluated the expression levels of klotho mRNA in isolated myofibers with their adjacent muscle stem cells isolated from young and old C57BL/6J mice (Fig. 6a). Interestingly, expression of klotho mRNA is reduced in myofibers with adjacent muscle stem cells from old mice directly after isolation and after $72 \mathrm{~h}$ of culture when the number of muscle stem cells on the myofibers is increased. This let us speculate that less sKlotho is secreted from aged myofibers and their adjacent muscle stem cells thereby impeding muscle stem cell function. To demonstrate that sKlotho can also inhibit canonical Wnt signaling in myogenic cells, we treated primary myoblasts with Wnt3a, sKlotho, or a combination of both (Fig. 6b). Importantly, Wnt3a-mediated activation of canonical Wnt signaling is inhibited by addition of sKlotho suggesting that sKlotho is also an inhibitor of canonical Wnt signaling in myogenic cells (Fig. 6b, Additional file 5: Figure S5A).

\section{Supplementation with sKlotho rejuvenates aged muscle stem cells}

We speculated that sKlotho could be one of the naturally occurring inhibitors of canonical Wnt signaling in muscle stem cells in the young and that loss of klotho expression in the aged might be one of the causes for increased canonical Wnt signaling in aged muscle stem cells. Therefore, we cultured muscle stem cells on floating fibers from young and old mice with different combinations of sKlotho protein (KL), Wnt3a and Dkk1, a well-known inhibitor of canonical Wnt signaling (Fig. 6c). Interestingly, we found that addition of sKlotho protein to fiber cultures obtained from old C57BL/6J mice (2224 months of age) resulted in an increase in the number of clusters per fiber formed after $72 \mathrm{~h}$ of culture and also an increased activation potential (Additional file 5: Figure S5C). Importantly, the number of clusters per fiber was similar to the number of clusters per fiber in young animals (4 months of age) suggesting that sKlotho rejuvenates old muscle stem cells (Fig. 6c). The effect of sKlotho on cluster numbers on aged fiber cultures was similar to the effect of Dkk1. Importantly, addition of sKlotho and Dkk1 to old myofibers did not lead to an increased cluster number compared to old myofibers treated with either sKlotho or Dkk1 alone indicating that sKlotho and Dkk1 are both acting by inhibition of canonical Wnt signaling (Additional file 5: Figure S5B). Addition of Wnt3a to young fibers resulted in a decrease in cluster numbers on young fibers as expected (Fig. 6c). Interestingly, the number of clusters was then similar to the ones on old myofibers. However, if we simultaneously added sKlotho and Wnt3a to the myofiber cultures from young mice, this sKlotho-mediated increase in the number of clusters per fiber was completely blocked suggesting that sKlotho and Wnt3a antagonize each other in muscle stem cells (Fig. 6d).

To address if sKlotho is also affecting self-renewal of muscle stem cells in the aged, we counted the number of Pax7-positive/MyoD-negative self-renewing muscle stem cells in clusters on myofibers of old and young mice treated with Wnt3a or sKlotho (Fig. 6e). sKlotho also increased the number of self-renewing muscle stem 
A
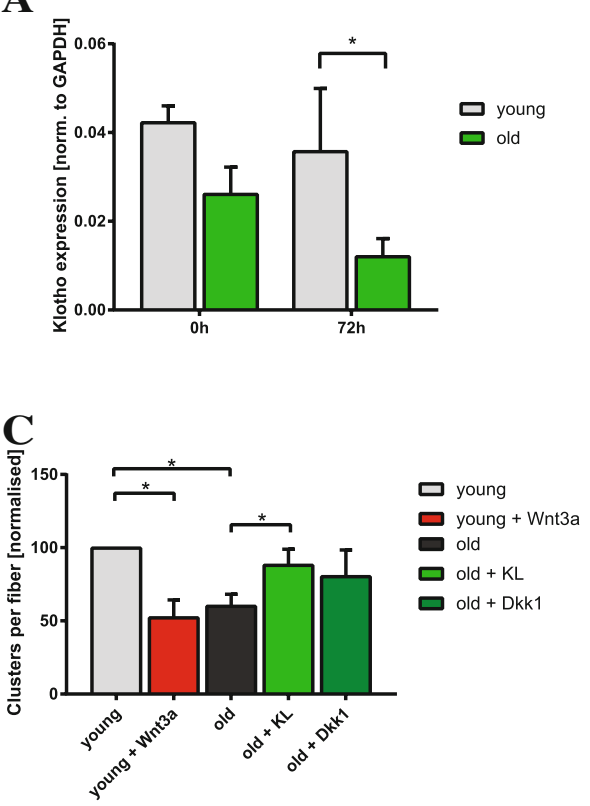

$\mathbf{E}$

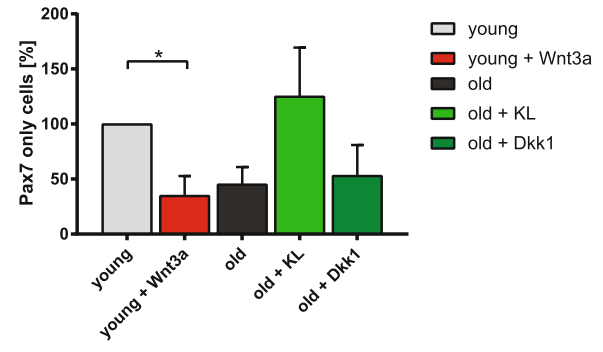

B

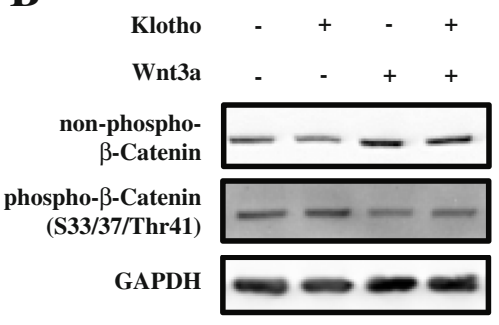

D
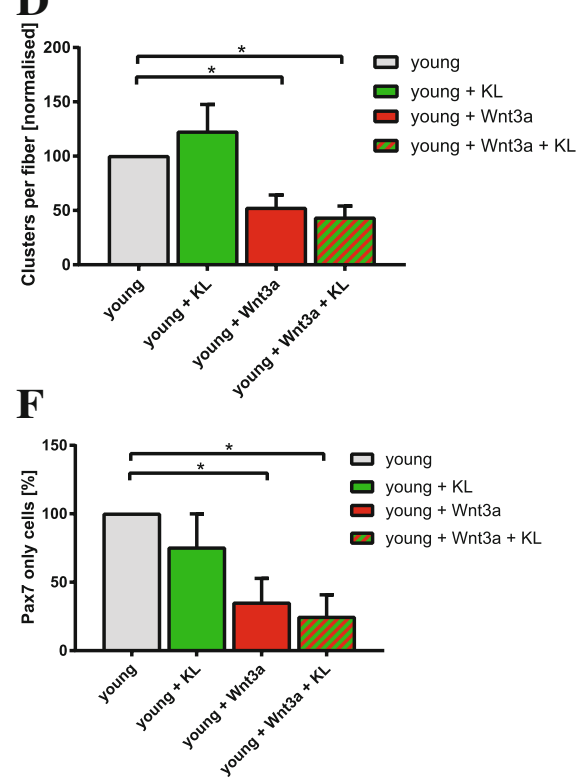

Fig. 6 sKlotho antagonizes aberrant Wnt3a function in aged muscle stem cells. a aKlotho mRNA expression in myofibers with their adjacent muscle stem cells directly after isolation or after $72 \mathrm{~h}$ of culture from young and old C57BL/6J mice (young mice: $n=4$, old mice: $n=5$ ). $\mathbf{b}$ Addition of sklotho to primary myoblasts reduces induction of canonical Wnt signaling evoked by addition of recombinant Wnt3a. c Myofibers with their adjacent muscle stem cells from young (4 months) and old (22-24 months) mice were cultured for $72 \mathrm{~h}$ with normal medium, recombinant soluble klotho (KL) protein, recombinant Wnt3a, or recombinant Dkk1. ( $n=6$ mice (young), $n=5$ (old)). The number of clusters per myofiber was normalized to young control. d Myofibers with their adjacent muscle stem cells from young (4 months) mice were cultured for $72 \mathrm{~h}$ with normal medium, recombinant soluble klotho (KL) protein, recombinant Wnt3a, or a combination of both ( $n=6$ mice). The number of clusters per myofiber was normalized to young control. e Addition of sKlotho recombinant protein (KL) increases the proportion of Pax7+/MyoDcells per fiber located in a cluster. The number of Pax7+/MyoD- cells (located in a cluster) per myofiber was normalized to young control ( $n=6$ mice (young), $n=5$ (old)). $\mathbf{f}$ Myofibers with their adjacent muscle stem cells from young (4 months) mice were cultured for $72 \mathrm{~h}$ with normal medium, recombinant soluble klotho $(\mathrm{KL})$ protein, recombinant Wnt3a, or a combination of both $(n=6$ mice). The number of Pax $7+/ \mathrm{MyoD}-$ cells (located in a cluster) per myofiber was normalized to young control. All data are presented as means \pm SEM. ${ }^{*} p<0.05,{ }^{* *} p<0.01,{ }^{* * *} p<0.001$

cells in the aged to numbers comparable to muscle stem cells on young fibers. Of note, Wnt3a and sKlotho are also counteracting each other regarding the self-renewal of young muscle stem cells (Fig. 6f) and a combined treatment with sKlotho and Dkk1 does not lead to increased self-renewal compared to treatment with either sKlotho or Dkk1 alone (Additional file 5: Figure S5D).

sKlotho might be an effective natural inhibitor of canonical Wnt signaling in muscle stem cells in the young and loss of klotho expression in the aged might be one of the causes for increased canonical Wnt signaling and thereby impeded muscle stem cell functionality in the aged. This, together with the data on supplementation of sKlotho in aged muscle stem cells, suggests that muscle stem cells and myofibers secrete sKlotho thereby inhibiting canonical Wnt signaling, a mechanism which is particularly hampered in the aged.

\section{Discussion}

Klotho hypomorphic mice display several aberrations in muscle function, for instance, reduced muscle strength and running endurance [24]. They also demonstrate reduced serum levels of sKlotho. Interestingly, a study by Semba et al. [44] found that reduced serum levels of sKlotho in older humans is correlated with reduced grip 
strength further strengthening the importance of constant klotho expression for maintenance and functional output of skeletal muscle. So far, nothing was known regarding effects of klotho expression on muscle stem cell function and differentiation of myoblasts.

Notably, in the current study, we observed that muscle stem cell function in klotho hypomorphic mice is massively perturbed. Furthermore, numbers of muscle stem cells are significantly decreased in $\Delta$ Klotho mice (Figs. 1 and $5 \mathrm{a}, \mathrm{c}$ ). Concomitantly, loss of klotho expression leads to a dramatic reduction in the regenerative capacity of skeletal muscle, which reminded us of the phenotype we observed during natural aging (Figs. 2 and 3; [7, 11]). Klotho hypomorphic mice are not only characterized by the loss of klotho gene expression but also by a disturbed mineral homeostasis caused by impaired FGF23 signaling in the kidney [45]. Since we could not rule out the effects of this disturbed mineral homeostasis in $\Delta$ Klotho mice, we performed experiments using cultures of muscle stem cells on single floating myofibers. In this system, we can exclude direct effects from the mineral homeostasis but not the fact that those changes might have led to changes in the epigenome or activity of signaling pathways before isolation. Such changes were described for naturally aged muscle stem cells [7, 11]. Of note, Wehling-Henricks and colleagues described that epigenetic silencing of klotho in muscular dystrophy contributes to the disturbed regeneration and fibrosis in mdx mice, the mouse model for $\mathrm{Du}$ chenne muscular dystrophy [46].

In our floating fiber culture system, we found that loss of klotho expression reduces the number of clusters formed and the number of cells per cluster suggesting that loss of klotho expression negatively influences proliferation and self-renewal of muscle stem cells (Fig. 5). Furthermore, differentiation of muscle stem cells on fibers is affected when expression of klotho is lost (Fig. 5 and Additional file 4: Figure S4) implying also a function of klotho in differentiation of muscle stem cells. In adipose-derived stem cells, loss of klotho expression hinders proliferation and differentiation [29]. Application of recombinant sKlotho protein on the other hand restored the proliferative capacity as well as the ability to differentiate. In the skin and intestine, loss of klotho results in exhaustion of the stem cell pool [42].

Canonical Wnt signaling plays an important role in muscle stem cell function [25]. Differentiation of muscle stem cells is controlled by canonical Wnt signaling, especially by signaling through the ligand Wnt3a. Although necessary for the differentiation process, aberrant canonical Wnt signaling results in increased fibrosis of skeletal muscle, for instance, in the context of aging or muscular dystrophy [40, 47]. Aged skeletal muscle has been shown to display increased canonical Wnt signaling resulting in a conversion of muscle stem cells from a myogenic to a fibrogenic lineage [40]. sKlotho is a known antagonist of canonical Wnt signaling [42]. Therefore we investigated if addition of sKlotho protein counteracts aberrant canonical Wnt signaling, here in the context of aging. Indeed, we found that Wnt3a antagonizes sKlotho function in myoblasts and old muscle stem cells (Fig. 6c-f). Furthermore we could demonstrate that inhibition of aberrant canonical Wnt signaling in the aged by Dkk1 had very similar effects compared to addition of sKlotho further supporting the notion that sKlotho is a naturally occurring inhibitor of canonical Wnt signaling in muscle stem cells.

We consider it likely that the large quantity of fibrotic tissue during impaired regeneration and the depletion of muscle stem cells in $\Delta$ Klotho mice can be traced back to chronic canonical Wnt stimulation as observed during natural aging [40]. Transforming growth factor beta (TFG- $\beta 1$ ) signaling is another pro-fibrotic pathway increased in skeletal muscle during aging resulting in reduced regenerative capacity [48]. Interestingly, sKlotho is a direct inhibitor of TGF- $\beta 1$ signaling by binding to the type-II TGF- $\beta$ receptor thereby preventing fibrosis [49]. Therefore, it is likely that sKlotho not only inhibits canonical Wnt signaling but also TFG- $\beta 1$ signaling in aged skeletal muscle thereby maintaining muscle stem cell function.

Importantly, restoration of sKlotho levels in the aged muscle stem cell niche improves muscle stem cell function in the aged. This suggests that acute sKlotho availability is dominant over intrinsic changes in aged muscle stem cells, which have been acquired over life time [7, 11, 12].

\section{Conclusions}

We conclude that reduction of sKlotho is an important contributor to loss of muscle stem cell function in the aged, most likely through loss of inhibition of canonical Wnt signaling. Therefore, replenishing the levels of sKlotho is a promising therapeutic approach for stimulation of muscle regeneration in the aged.

\section{Additional files}

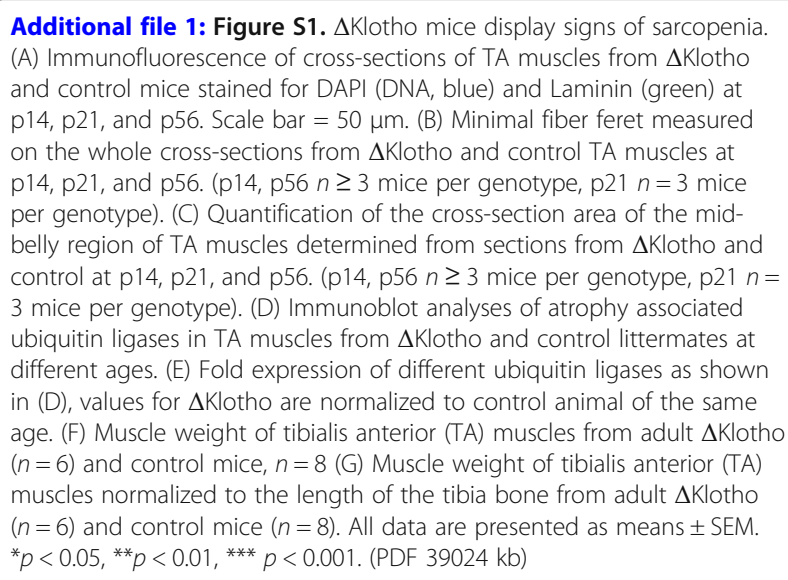


Additional file 2: Figure S2. Fiber types are not changed in $\Delta$ Klotho mice. (A) Representative immunofluorescence stainings of cross-sections from EDL of p56 $\Delta$ Klotho and control mice stained for DAPI (DNA, blue), laminin (white), MHClla and MHCllb, or MHClla and MHCllx, respectively. Scale bar $=50 \mu \mathrm{m}$. (B) Percentage of MHC type lla and Ilb positive myofibers on EDL cross-sections from $\Delta$ Klotho and control mice at p56. ( $\Delta$ Klotho $n=3$ mice, control $n=7$ mice). (C) Percentage of MHC type lla and IIx positive myofibers on EDL cross-sections from $\Delta$ Klotho and control mice at p56. ( $\Delta$ Klotho $n=3$ mice, control $n=6$ mice). All data are presented as means \pm SEM. (PDF $8407 \mathrm{~kb}$ )

Additional file 3: Figure S3. The differentiation of myoblasts from $\Delta$ Klotho mice is not affected in vitro. (A) Quantification of the average number of nuclei per myotube counted on 6 random regions of interest per condition after 5 days of differentiation ( $\Delta$ Klotho $n=3$ mice, control $n=$ 4 mice). (B) Percentage of myogenin-positive nuclei of all nuclei within myotubes after 5 days of differentiation ( $\Delta$ Klotho $n=3$ mice, control $n=4$ mice) (C) Differentiation index (percentage of myotubes with more than three nuclei) after 5 days of differentiation ( $\Delta$ Klotho $n=3$ mice, control $n=4$ mice). (D) Distribution of classes of myotubes after 5 days of differentiation ( $\Delta$ Klotho $n=3$ mice, control $n=4$ mice). (E) Immunoblot analyses of supernatants from primary myoblasts and lysate from a kidney from wt animals using an antibody directed against klotho showing sKlotho in the supernatant and in whole kidney lysates (as expected). (F) Ponceau stained membrane showing similar loading of concentrated supernatants from primary myoblasts isolated from $\Delta$ Klotho and control mice. All data are presented as means \pm SEM. (PDF $7378 \mathrm{~kb}$ )

Additional file 4: Figure S4. Muscle stem cell function is impaired in adult $\Delta$ Klotho mice. (A) The activation potential (number of clusters per myofiber after $72 \mathrm{~h}$ of culture divided by the number of muscle stem cells per myofiber directly after isolation) ( $\Delta$ Klotho $n=5$ mice, control $n$ $=7$ mice). (B) Percentage of Pax7+/MyoD- cells within a cluster on myofibers isolated from p42 old mice. ( $\Delta$ Klotho $n=5$ mice, control $n=7$ mice). (C) Percentage of Pax7+/MyoD+ cells within a cluster on myofibers isolated from p42 old mice. ( $\Delta$ Klotho $n=5$ mice, control $n=7$ mice). (D) Percentage of Pax7-/MyoD- cells within clusters on myofibers isolated from p42 old mice. ( $\Delta$ Klotho $n=5$ mice, control $n=7$ mice). All data are presented as means \pm SEM. ${ }^{*} p<0.05$. (PDF $511 \mathrm{~kb}$ )

Additional file 5: Figure S5. Addition of recombinant sKlotho protein rejuvenates aged muscle stem cells. (A) Addition of sKlotho to primary myoblasts reduces canonical Wnt signaling induced by addition of recombinant Wnt3a as evidenced by measuring levels of phosho-betacatenin and non-phospho-beta-catenin, densitometric analysis of Fig. 6b after normalization to GAPDH, values are shown as normalization to control. (B) Myofibers with their adjacent muscle stem cells from young ( 4 months) and old (22-24 months) mice were cultured for $72 \mathrm{~h}$ with medium, recombinant soluble klotho (KL) protein, recombinant Dkk1 or a combination of both. The number of clusters per myofiber was normalized to young control. ( $n=4$ mice (young), $n=3$ (old)). (C) The activation potential is increased in old mice when sKlotho protein $(\mathrm{KL})$ is added $(n$ =4). (D) Myofibers with their adjacent muscle stem cells from young (4 months) and old (22-24 months) mice were cultured for $72 \mathrm{~h}$ with medium, recombinant soluble klotho $(\mathrm{KL})$ protein, recombinant $\mathrm{Dkk} 1$ or a combination of both. The number of Pax7+/MyoD- cells per myofiber was normalized to young control. ( $n=4$ mice (young), $n=3$ (old)). All data are presented as means \pm SEM. ${ }^{*} p<0.05$. (PDF $616 \mathrm{~kb}$ )

\section{Abbreviations}

devMHC: Developmental myosin heavy chain; Dkk1: Dickkopf-related protein 1; EDL: Extensor digitorum longum; Fbxo32: F-Box Protein 32;

FGF23: Fibroblast growth factor 23; Hox: Homeobox; IGF1: Insulin-like growth factor 1; JAK: Janus kinase; mKlotho: Membrane Klotho; MuRF: Muscle RINGfinger protein; MyoD: Myogenic differentiation 1; Pax7: Paired box protein 7; sKlotho: Soluble Klotho; SPF: Specific pathogen free; STAT: Signal Transducer and Activator of Transcription proteins; TA: Tibialis anterior; TGF$\beta$ : Transforming growth factor $\beta$; Wnt: Wingless-related integration site

\section{Acknowledgements}

We thank Christine Poser, Christina Picker, Sabine Landmann, and Daniela Reichenbach for their excellent technical assistance and the FLI Core
Facilities histology (L. Rothenburger) and imaging and the mouse facility (J. Brüchert, C. Maisch) for their services. We thank M. Kuro-o for providing the klotho hypomorphic mice.

\section{Funding}

J.V.M. and M.S. were supported by a grant from the German Research Foundation (DFG, MA-3975/2-1). HEA was supported by a grant from the Leibniz association (SAW2015)

\section{Availability of data and materials}

All datasets generated and analyzed during the current study are available from the corresponding author upon reasonable request.

\section{Authors' contributions}

HEA and JH performed most experiments, analyzed the data, and interpreted the results. MS performed the GPCR and immunoblot analyses. HEA and JVM designed and performed the experiments, analyzed the data, interpreted the results, and wrote the manuscript. CK provided support and suggestions for the experiments concerning klotho hypomorphic mice. All authors read and approved the final version of the manuscript.

\section{Ethics approval}

All animal experiments were performed in accordance with the German Animal Welfare Act and approved by the responsible local authority of Thuringia (TLV), TVA no.: 03-11/14.

\section{Competing interests}

The authors declare that they have no competing interest.

\section{Publisher's Note}

Springer Nature remains neutral with regard to jurisdictional claims in published maps and institutional affiliations.

\section{Author details}

'Leibniz-Institute on Aging - Fritz-Lipmann-Institute, Beutenbergstrasse 11, 07745 Jena, Germany. ${ }^{2}$ Present address: Max-Delbrück-Center for Molecular Medicine, Robert-Rössle-Str. 10, 13125 Berlin, Germany.

Received: 29 January 2018 Accepted: 21 June 2018

Published online: 04 July 2018

\section{References}

1. Bentzinger CF, von Maltzahn J, Rudnicki MA. Extrinsic regulation of satellite cell specification. Stem Cell Res Ther. 2010;1:27.

2. Lepper C, Partridge TA, Fan CM. An absolute requirement for Pax7-positive satellite cells in acute injury-induced skeletal muscle regeneration. Development. 2011;138:3639-46.

3. Murphy MM, Lawson JA, Mathew SJ, Hutcheson DA, Kardon G. Satellite cells, connective tissue fibroblasts and their interactions are crucial for muscle regeneration. Development. 2011;138:3625-37.

4. Bentzinger CF, Wang YX, Dumont NA, Rudnicki MA. Cellular dynamics in the muscle satellite cell niche. EMBO Rep. 2013;14:1062-72.

5. von Maltzahn J, Bentzinger CF, Rudnicki MA. Characteristics of satellite cells and multipotent adult stem cells in the skeletal muscle. Dordrecht: Springer; 2013.

6. Dumont NA, Wang YX, von Maltzahn J, Pasut A, Bentzinger CF, Brun CE, Rudnicki MA. Dystrophin expression in muscle stem cells regulates their polarity and asymmetric division. Nat Med. 2015;21:1455-63.

7. Price FD, von Maltzahn J, Bentzinger CF, Dumont NA, Yin H, Chang NC, Wilson DH, Frenette J, Rudnicki MA. Inhibition of JAK-STAT signaling stimulates adult satellite cell function. Nat Med. 2014;20:1174-81.

8. Sousa-Victor P, Garcia-Prat L, Serrano AL, Perdiguero E, Munoz-Canoves P. Muscle stem cell aging: regulation and rejuvenation. Trends Endocrinol Metab. 2015;26:287-96.

9. Chakkalakal JV, Jones KM, Basson MA, Brack AS. The aged niche disrupts muscle stem cell quiescence. Nature. 2012;490:355-60.

10. Lukjanenko L, Jung MJ, Hegde N, Perruisseau-Carrier C, Migliavacca E, Rozo M, Karaz S, Jacot G, Schmidt M, Li L, Metairon S, Raymond F, Lee U, Sizzano F, Wilson DH, Dumont NA, Palini A, Fassler R, Steiner P, Descombes P, Rudnicki MA, Fan CM, von Maltzahn J, Feige JN, Bentzinger CF. Loss of fibronectin from the aged stem cell niche affects the regenerative capacity of skeletal muscle in mice. Nat Med. 2016;22:897-905. 
11. Schworer S, Becker F, Feller C, Baig AH, Kober U, Henze H, Kraus JM, Xin B, Lechel A, Lipka DB, Varghese CS, Schmidt M, Rohs R, Aebersold R, Medina KL, Kestler HA, Neri F, von Maltzahn J, Tumpel S, Rudolph KL. Epigenetic stress responses induce muscle stem-cell ageing by Hoxa9 developmental signals. Nature. 2016;540:428-32.

12. Sousa-Victor P, Gutarra S, Garcia-Prat L, Rodriguez-Ubreva J, Ortet L, RuizBonilla V, Jardi M, Ballestar E, Gonzalez S, Serrano AL, Perdiguero E, Munoz Canoves P. Geriatric muscle stem cells switch reversible quiescence into senescence. Nature. 2014;506:316-21.

13. $\mathrm{Xu}$ Y, Sun Z. Molecular basis of Klotho: from gene to function in aging Endocr Rev. 2015:36:174-93.

14. Bian A, Neyra JA, Zhan M, Hu MC. Klotho, stem cells, and aging. Clin Interv Aging. 2015;10:1233-43.

15. Kurosu H, Ogawa Y, Miyoshi M, Yamamoto M, Nandi A, Rosenblatt KP, Baum MG, Schiavi S, Hu MC, Moe OW, Kuro-o M. Regulation of fibroblast growth factor-23 signaling by klotho. J Biol Chem. 2006;281:6120-3.

16. Quarles LD. Endocrine functions of bone in mineral metabolism regulation. J Clin Invest. 2008;118:3820-8.

17. Utsugi $T$, Ohno T, Ohyama $Y$, Uchiyama T, Saito $Y$, Matsumura $Y$, Aizawa $H$, Itoh H, Kurabayashi M, Kawazu S, Tomono S, Oka Y, Suga T, Kuro-o M, Nabeshima Y, Nagai R. Decreased insulin production and increased insulin sensitivity in the klotho mutant mouse, a novel animal model for human aging. Metabolism. 2000;49:1118-23.

18. Abramovitz L, Rubinek T, Ligumsky H, Bose S, Barshack I, Avivi C, Kaufman B, Wolf I. KL1 internal repeat mediates klotho tumor suppressor activities and inhibits bFGF and IGF-I signaling in pancreatic cancer. Clin Cancer Res. 2011 17:4254-66.

19. Pedersen L, Pedersen SM, Brasen CL, Rasmussen LM. Soluble serum Klotho levels in healthy subjects. Comparison of two different immunoassays. Clin Biochem. 2013:46:1079-83.

20. Sato S, Kawamata Y, Takahashi A, Imai Y, Hanyu A, Okuma A, Takasugi M, Yamakoshi K, Sorimachi H, Kanda H, Ishikawa Y, Sone S, Nishioka Y, Ohtani N, Hara E. Ablation of the p16(INK4a) tumour suppressor reverses ageing phenotypes of klotho mice. Nat Commun. 2015;6:7035.

21. Kuro-o M, Matsumura Y, Aizawa H, Kawaguchi H, Suga T, Utsugi T, Ohyama Y, Kurabayashi M, Kaname T, Kume E, Iwasaki H, lida A, Shiraki-lida T, Nishikawa S, Nagai R, Nabeshima YI. Mutation of the mouse klotho gene leads to a syndrome resembling ageing. Nature. 1997;390:45-51.

22. Kurosu H, Yamamoto M, Clark JD, Pastor JV, Nandi A, Gurnani P, McGuinness OP, Chikuda H, Yamaguchi M, Kawaguchi H, Shimomura I, Takayama Y, Herz J, Kahn CR, Rosenblatt KP, Kuro-o M. Suppression of aging in mice by the hormone Klotho. Science. 2005:309:1829-33.

23. Olauson $H$, Mencke R, Hillebrands JL, Larsson TE. Tissue expression and source of circulating alphaKlotho. Bone. 2017;100:19-35.

24. Phelps M, Pettan-Brewer C, Ladiges W, Yablonka-Reuveni Z. Decline in muscle strength and running endurance in klotho deficient C57BL/6 mice. Biogerontology. 2013;14:729-39.

25. von Maltzahn J, Chang NC, Bentzinger CF, Rudnicki MA. Wnt signaling in myogenesis. Trends Cell Biol. 2012;22:602-9.

26. von Maltzahn J, Jones AE, Parks RJ, Rudnicki MA. Pax7 is critical for the normal function of satellite cells in adult skeletal muscle. Proc Natl Acad Sci U S A. 2013;110:16474-9.

27. von Maltzahn J, Zinoviev R, Chang NC, Bentzinger CF, Rudnicki MA. A truncated Wnt7a retains full biological activity in skeletal muscle. Nat Commun. 2013:4:2869.

28. Briguet A, Courdier-Fruh I, Foster M, Meier T, Magyar JP. Histological parameters for the quantitative assessment of muscular dystrophy in the mdx-mouse. Neuromuscul Disord. 2004;14:675-82.

29. Fan J, Sun Z. The antiaging gene klotho regulates proliferation and differentiation of adipose-derived stem cells. Stem Cells. 2016;34:1615-25.

30. Rom O, Reznick AZ. The role of E3 ubiquitin-ligases MuRF-1 and MAFbx in loss of skeletal muscle mass. Free Radic Biol Med. 2016:98:218-30.

31. Gumucio JP, Mendias CL. Atrogin-1, MuRF-1, and sarcopenia. Endocrine. 2013:43:12-21.

32. Bodine SC, Latres E, Baumhueter S, Lai VK, Nunez L, Clarke BA, Poueymirou WT, Panaro FJ, Na E, Dharmarajan K, Pan ZQ, Valenzuela DM, DeChiara TM, Stitt TN, Yancopoulos GD, Glass DJ. Identification of ubiquitin ligases required for skeletal muscle atrophy. Science. 2001;294: 1704-8
33. Gomes MD, Lecker SH, Jagoe RT, Navon A, Goldberg AL. Atrogin-1, a muscle-specific F-box protein highly expressed during muscle atrophy. Proc Natl Acad Sci U S A. 2001;98:14440-5.

34. Bentzinger CF, Wang YX, Rudnicki MA. Building muscle: molecular regulation of myogenesis. Cold Spring Harb Perspect Biol. 2012;4:1-16.

35. Schiaffino S, Rossi AC, Smerdu V, Leinwand LA, Reggiani C. Developmental myosins: expression patterns and functional significance. Skelet Muscle. 2015:5:22.

36. Pasut A, Oleynik P, Rudnicki MA. Isolation of muscle stem cells by fluorescence activated cell sorting cytometry. Methods Mol Biol. 2012;798:53-64.

37. Zammit PS, Golding JP, Nagata Y, Hudon V, Partridge TA, Beauchamp JR. Muscle satellite cells adopt divergent fates: a mechanism for self-renewal? J Cell Biol. 2004;166:347-57.

38. Cooper RN, Tajbakhsh S, Mouly V, Cossu G, Buckingham M, Butler-Browne GS. In vivo satellite cell activation via Myf5 and MyoD in regenerating mouse skeletal muscle. J Cell Sci. 1999;112 ( Pt 17:2895-901.

39. Ring A, Kim YM, Kahn M. Wnt/catenin signaling in adult stem cell physiology and disease. Stem Cell Rev. 2014;10:512-25.

40. Brack AS, Conboy MJ, Roy S, Lee M, Kuo CJ, Keller C, Rando TA. Increased Wnt signaling during aging alters muscle stem cell fate and increases fibrosis. Science. 2007;317:807-10.

41. Brack AS, Conboy IM, Conboy MJ, Shen J, Rando TA. A temporal switch from notch to Wnt signaling in muscle stem cells is necessary for normal adult myogenesis. Cell Stem Cell. 2008;2:50-9.

42. Liu H, Fergusson MM, Castilho RM, Liu J, Cao L, Chen J, Malide D, Rovira D II, Schimel CJ, Kuo JS, Gutkind PM, Hwang TF. Augmented Wnt signaling in a mammalian model of accelerated aging. Science. 2007;317:803-6.

43. Mirza SB, Ekhteiari Salmas R, Fatmi MQ, Durdagi S. Discovery of Klotho peptide antagonists against Wnt3 and Wnt3a target proteins using combination of protein engineering, protein-protein docking, peptide docking and molecular dynamics simulations. J Enzyme Inhib Med Chem. 2017;32:84-98

44. Semba RD, Cappola AR, Sun K, Bandinelli S, Dalal M, Crasto C, Guralnik JM, Ferrucci L. Relationship of low plasma klotho with poor grip strength in older community-dwelling adults: the InCHIANTI study. Eur J Appl Physiol. 2012;112:1215-20.

45. Kuro-o M. Klotho as a regulator of fibroblast growth factor signaling and phosphate/calcium metabolism. Curr Opin Nephrol Hypertens. 2006;15:437-41.

46. Wehling-Henricks M, Li Z, Lindsey C, Wang Y, Welc SS, Ramos JN, Khanlou $\mathrm{N}$, Kuro OM, Tidball JG. Klotho gene silencing promotes pathology in the mdx mouse model of Duchenne muscular dystrophy. Hum Mol Genet. 2016:25:2465-82.

47. Trensz F, Haroun S, Cloutier A, Richter MV, Grenier G. A muscle resident cell population promotes fibrosis in hindlimb skeletal muscles of mdx mice through the Wnt canonical pathway. Am J Phys Cell Physiol. 2010;299:C939-47.

48. Carlson ME, Hsu M, Conboy IM. Imbalance between pSmad3 and Notch induces CDK inhibitors in old muscle stem cells. Nature. 2008;454:528-32.

49. Doi S, Zou Y, Togao O, Pastor JV, John GB, Wang L, Shiizaki K, Gotschall R, Schiavi S, Yorioka N, Takahashi M, Boothman DA, Kuro-o M. Klotho inhibits transforming growth factor-beta1 (TGF-beta1) signaling and suppresses renal fibrosis and cancer metastasis in mice. J Biol Chem. 2011;286:8655-65.

\section{Ready to submit your research? Choose BMC and benefit from:}

- fast, convenient online submission

- thorough peer review by experienced researchers in your field

- rapid publication on acceptance

- support for research data, including large and complex data types

- gold Open Access which fosters wider collaboration and increased citations

- maximum visibility for your research: over $100 \mathrm{M}$ website views per year

At BMC, research is always in progress.

Learn more biomedcentral.com/submissions 